Homology, Homotopy and Applications, vol.20(1), 2018, pp.69-78

\title{
GOLODNESS AND POLYHEDRAL PRODUCTS OF SIMPLICIAL COMPLEXES WITH MINIMAL TAYLOR RESOLUTIONS
}

\author{
KOUYEMON IRIYE AND DAISUKE KISHIMOTO
}

(communicated by Donald M. Davis)

\begin{abstract}
Let $K$ be a simplicial complex such that the Taylor resolution for its Stanley-Reisner ring is minimal. We prove that the following conditions are equivalent: (1) $K$ is Golod; (2) any two minimal non-faces of $K$ are not disjoint; (3) the moment-angle complex for $K$ is homotopy equivalent to a wedge of spheres; (4) the decomposition of the suspension of the polyhedral product $\mathcal{Z}_{K}(C \underline{X}, \underline{X})$ due to Bahri, Bendersky, Cohen and Gitler desuspends.
\end{abstract}

\section{Introduction}

Golodness is a property of a graded commutative $\operatorname{ring} R$ which is originally defined by a certain equality involving a Poincaré series of the cohomology of $R$, and Golod [6] gave an equivalent condition in terms of the derived torsion algebra of $R$. Golodness has been intensively studied for Stanley-Reisner rings since those of important simplicial complexes such as dual sequentially Cohen-Macaulay complexes are known to have the Golod property, and in this paper, we are interested in Golodness of Stanley-Reisner rings. So we here define Golodness of Stanley-Reisner rings in terms of their derived torsion algebras. Let $K$ be a simplicial complex on the vertex set $[m]:=\{1, \ldots, m\}$, and let $\mathbb{k}$ be a commutative ring. Recall that the Stanley-Reisner ring of $K$ over $\mathbb{k}$ is defined by

$$
\mathbb{k}[K]:=\mathbb{k}\left[v_{1}, \ldots, v_{m}\right] /\left(v_{I} \mid I \subset[m], I \notin K\right),
$$

where $\left|v_{i}\right|=2$ and $v_{I}=v_{i_{1}} \cdots v_{i_{k}}$ for $I=\left\{i_{1}, \ldots, i_{k}\right\}$. We consider the derived algebra $\operatorname{Tor}_{\mathbb{k}\left[v_{1}, \ldots, v_{m}\right]}(\mathbb{k}[K], \mathbb{k})$ and fix its products and (higher) Massey products to those induced from the Koszul resolution of $\mathbb{k}$ over $\mathbb{k}\left[v_{1}, \ldots, v_{m}\right]$ tensored with $\mathbb{k}[K]$. Let $R^{+}$denote the positive degree part of a graded ring $R$.

Definition 1.1. The Stanley-Reisner ring $\mathbb{k}[K]$ is called Golod if all products and (higher) Massey products in $\operatorname{Tor}_{\mathbb{k}\left[v_{1}, \ldots, v_{m}\right]}^{+}(\mathbb{k}[K], \mathbb{k})$ are trivial.

K.I. is supported by JSPS KAKENHI (No. 26400094), and D.K. is supported by JSPS KAKENHI (No. 25400087).

Received May 9, 2017, revised August 22, 2017; published on December 27, 2017.

2010 Mathematics Subject Classification: 13F55, 55P15.

Key words and phrases: Stanley-Reisner ring, Golod property, Taylor resolution, polyhedral product, fat wedge filtration.

Article available at http://dx.doi.org/10.4310/HHA.2018.v20.n1.a5

Copyright (C) 2017, International Press. Permission to copy for private use granted. 
One of the biggest problems in Golodness of Stanley-Reisner rings is to get a combinatorial characterization of Golodness, where we have many examples of interesting simplicial complexes. This is still open at this moment while there have been many attempts. Then we consider the following weaker problem.

Problem 1.2. Find a class of simplicial complexes for which Golodness of StanleyReisner rings can be combinatorially characterized.

In a seminal paper [4], Davis and Januszkiewicz showed that the cohomology with coefficients $\mathbb{k}$ of a certain space constructed from a simplicial complex $K$, called the Davis-Januszkiewicz space for $K$, is isomorphic to the Stanley-Reisner ring $\mathbb{k}[K]$. This opens a way of a topological study of Stanley-Reisner rings. Moreover, Baskakov, Buchstaber and Panov [3] found an isomorphism between the cohomology with coefficients $\mathbb{k}$ of the space $\mathcal{Z}_{K}$, called the moment-angle complex for $K$, and the derived torsion algebra $\operatorname{Tor}_{\mathbb{k}\left[v_{1}, \ldots, v_{m}\right]}^{*}(\mathbb{k}[K], \mathbb{k})$ which respects products and (higher) Massey products. Then we can study Golodness of Stanley-Reisner rings by investigating the homotopy types of moment-angle complexes. Thus there is a trinity in studying Golodness of Stanley-Reisner rings consisting of algebra, combinatorics and homotopy theory.

In this paper, we consider Problem 1.2 under the above trinity, and we will prove the following, where the notation in the condition 4 will be defined later. Recall that a non-empty subset $N$ of the vertex set of a simplicial complex $K$ is a minimal non-face if $N \notin K$ and $N-i \in K$ whenever $i \in N$. Put $[m]:=\{1, \ldots, m\}$.

Theorem 1.3. Let $K$ be a simplicial complex on the vertex set $[m]$ such that $\mathbb{k}[K]$ has a minimal Taylor resolution. Then the following conditions are equivalent:

1. $\mathbb{k}[K]$ is Golod;

2. any two minimal non-faces of $K$ are not disjoint;

3. the moment-angle complex for $K$ is homotopy equivalent to a wedge of spheres;

4. for any $\underline{X}=\left\{X_{i}\right\}_{i=1}^{m}$, there is a homotopy decomposition of a polyhedral product

$$
\mathcal{Z}_{K}(C \underline{X}, \underline{X}) \simeq \bigvee_{\emptyset \neq I \subset[m]}\left|\Sigma K_{I}\right| \wedge \widehat{X}^{I}
$$

Remark 1.4. 1. In Theorem 1.3, Golodness does not depend on the ground ring, but in general, this is not true as in $[\mathbf{1 0}, \mathbf{8}]$. We will see in the next section that minimality of the Taylor resolution of $\mathbb{k}[K]$ does not depend on $\mathbb{k}$, so in fact, Theorem 1.3 does not depend on $\mathbb{k}$.

2. Recently, Frankhuizen [5] proved the equivalence between 1 and 2 in a more general setting by a purely algebraic manner.

Throughout this paper, let $K$ denote a simplicial complex on the vertex set $[m]$, where $K$ might have ghost vertices.

\section{Minimality of the Taylor resolutions}

In this section, we recall the definition of the Taylor resolution for a Stanley-Reisner ring and a combinatorial characterization of its minimality due to Ayzenberg [1]. We then prove the implication $1 \Rightarrow 2$ of Theorem 1.3. 
Let $N_{1}, \ldots, N_{r}$ be minimal non-faces of $K$. Then we have

$$
\mathbb{k}[K]=\mathbb{k}\left[v_{1}, \ldots, v_{m}\right] /\left(v_{N_{1}}, \ldots, v_{N_{r}}\right) .
$$

The Taylor resolution for $\mathbb{k}[K]$ is the free $\mathbb{k}\left[v_{1}, \ldots, v_{m}\right]$-module resolution

$$
\cdots \stackrel{d}{\rightarrow} R^{-\ell} \stackrel{d}{\rightarrow} R^{-\ell+1} \stackrel{d}{\rightarrow} \cdots \stackrel{d}{\rightarrow} R^{0}=\mathbb{k}\left[v_{1}, \ldots, v_{m}\right] \stackrel{\text { proj }}{\rightarrow} \mathbb{k}[K],
$$

such that $R^{-\ell}$ is the free $\mathbb{k}\left[v_{1}, \ldots, v_{m}\right]$-module generated by symbols $w_{i_{1}, \ldots, i_{\ell}}$ for $1 \leqslant i_{1}<\cdots<i_{\ell} \leqslant r$ with the differential

$$
d\left(w_{i_{1}, \ldots, i_{\ell}}\right)=\sum_{k=1}^{\ell}(-1)^{k+1} v_{N_{i_{k}}-N_{i_{1}} \cup \ldots \cup \widehat{N_{i_{k}}} \cup \ldots \cup N_{i_{\ell}}} w_{i_{1}, \ldots, \widehat{i_{k}}, \ldots, i_{\ell}},
$$

where we set $v_{\emptyset}=1$. As usual, we say that the Taylor resolution is minimal if the differential satisfies

$$
d \otimes_{\mathbb{k}\left[v_{1}, \ldots, v_{m}\right]} \mathbb{k}=0 .
$$

By definition, minimality of the Taylor resolution for $\mathbb{k}[K]$ does not depend on the ground ring $\mathbb{k}$, so we say that $K$ has a minimal Taylor resolution if the Taylor resolution for $\mathbb{k}[K]$ is minimal for some $\mathbb{k}$. Minimality of the Taylor resolution for $\mathbb{k}[K]$ can be readily translated combinatorially as:

Proposition 2.1 (Ayzenberg [1]). Let $N_{1}, \ldots, N_{r}$ be minimal non-faces of $K$. Then $K$ has a minimal Taylor resolution if and only if

$$
N_{i} \not \subset \bigcup_{k \neq i} N_{k} \quad \text { for all } i .
$$

From any given simplicial complex, Ayzenberg [1] constructed a new simplicial complex with a minimal Taylor resolution, and here we generalize his construction. Let $\mathrm{N}=\left\{N_{1}, \ldots, N_{r}\right\}$ be a sequence of subsets of a finite set $W$, where we allow $N_{i}=N_{j}$ for some $i \neq j$ and call $W$ the ground set of $\mathrm{N}$. We allow $\mathrm{N}$ to be empty. By introducing new distinct points $a_{1}, \ldots, a_{r}$, we put $\widetilde{N}_{i}=N_{i} \sqcup\left\{a_{i}\right\}$ and $V=W \sqcup$ $\left\{a_{1}, \ldots, a_{r}\right\}$. Define $K(\mathrm{~N})$ to be the simplicial complex on the vertex set $V$ whose minimal non-faces are $\widetilde{N}_{1}, \ldots, \widetilde{N}_{r}$. When $\mathrm{N}$ is empty, we have $K(\mathrm{~N})=\Delta^{W}$, where $\Delta^{W}$ denotes the full simplex on the vertex set $W$. We further translate Proposition 2.1 in terms of the simplicial complex $K(\mathrm{~N})$, which will be used in Section 4 below.

Proposition 2.2. A simplicial complex $K$ has a minimal Taylor resolution if and only if there is a sequence $\mathrm{N}$ of subsets of a finite set $W$ such that $K \cong K(\mathrm{~N})$.

Proof. Since $\widetilde{N}_{i} \not \subset \bigcup_{k \neq i} \widetilde{N}_{k}$ for all $i, K(\mathrm{~N})$ has a minimal Taylor resolution by Proposition 2.1. Then $K$ has a minimal Taylor resolution whenever $K \cong K(\mathrm{~N})$. Suppose next that $K$ has a minimal Taylor resolution. Let $N_{1}, \ldots, N_{r}$ be all minimal non-faces of $K$. By Proposition 2.1 there exists $a_{i} \in N_{i}-\bigcup_{k \neq i} N_{k}$ for all $i$, where $a_{1}, \ldots, a_{r}$ are distinct. Put $W:=[m]-\left\{a_{1}, \ldots, a_{r}\right\}$. If we put $\mathrm{N}=\left\{N_{1}-a_{1}, \ldots, N_{r}-a_{r}\right\}$ which is a sequence of subsets of $W$, we have $K=K(\mathrm{~N})$ as desired.

We prove the implication $1 \Rightarrow 2$ of Theorem 1.3. For this, we use the following lemma, the proof of which will be given in the next section. For a subset $I \subset[m]$, we put

$$
K_{I}:=\{\sigma \in K \mid \sigma \subset I\}
$$


Lemma 2.3. If $K_{I \cup J}=\partial \Delta^{I} * \partial \Delta^{J}$ for some non-empty $I, J \subset[m]$ with $I \cap J=\emptyset$, then $K$ is not Golod.

Next we record an obvious fact of minimal non-faces, where we omit the proof. For a simplex $\sigma \in K$, let $\operatorname{lk}_{K}(\sigma)$ denote the link of $\sigma$ in $K$.

Lemma 2.4. Let $N_{1}, \ldots, N_{r}$ be minimal non-faces of $K$.

1. For a simplex $\sigma \in K$, any minimal non-face of $\operatorname{lk}_{K}(\sigma)$ has the form $N_{i}-\sigma$ for some $i$.

2. For a subset $I \subset[m]$, minimal non-faces of $K_{I}$ are $N_{i}$ 's with $N_{i} \subset I$.

Proposition 2.5. Suppose $K$ has a minimal Taylor resolution. If $\mathbb{k}[K]$ is Golod, then any two minimal non-faces of $K$ are not disjoint.

Proof. Let $N_{1}, \ldots, N_{r}$ be minimal non-faces of $K$. Assume $N_{i} \cap N_{j}=\emptyset$ for some $i \neq j$. By Proposition 2.1, we have $N_{k} \not \subset N_{i} \cup N_{j}$ for any $k \neq i, j$. Then by Lemma 2.4, $N_{i}, N_{j}$ are the only minimal non-faces of $K_{N_{i} \cup N_{j}}$. It follows that $K_{N_{i} \cup N_{j}}=\partial \Delta^{N_{i}} *$ $\partial \Delta^{N_{j}}$. Then we have $\left|N_{i}\right| \geqslant 1$ and $\left|N_{j}\right| \geqslant 1$. Thus by Lemma 2.3, $K$ is not Golod, completing the proof.

\section{Polyhedral products}

In this section, we recall the definition of polyhedral products and their properties that we are going to use. Let $(\underline{X}, \underline{A})=\left\{\left(X_{i}, A_{i}\right)\right\}_{i \in[m]}$ be a sequence of pairs of spaces indexed by vertices of $K$. The polyhedral product $\mathcal{Z}_{K}(\underline{X}, \underline{A})$ is defined by

$$
\mathcal{Z}_{K}(\underline{X}, \underline{A}):=\bigcup_{\sigma \in K}(\underline{X}, \underline{A})^{\sigma} \quad\left(\subset X_{1} \times \cdots \times X_{m}\right),
$$

where

$$
(\underline{X}, \underline{A})^{\sigma}=Y_{1} \times \cdots \times Y_{m} \quad \text { for } \quad Y_{i}= \begin{cases}X_{i} & i \in \sigma, \\ A_{i} & i \notin \sigma .\end{cases}
$$

For a sequence of pointed spaces $\underline{X}=\left\{X_{i}\right\}_{i \in[m]}$, we put $(C \underline{X}, \underline{X}):=\left\{\left(C X_{i}, X_{i}\right)\right\}_{i \in[m]}$, where $C Y$ denotes the reduced cone of a pointed space $Y$. The real moment-angle complex $\mathbb{R} \mathcal{Z}_{K}$ is the polyhedral product $\mathcal{Z}_{K}(C \underline{X}, \underline{X})$ with $X_{i}=S^{0}$ for all $i$ while the moment-angle complex $\mathcal{Z}_{K}$ is $\mathcal{Z}_{K}(C \underline{X}, \underline{X})$ with $X_{i}=S^{1}$ for any $i$. Recall from [7] that the fat wedge filtration

$$
*=\mathcal{Z}_{K}^{0}(C \underline{X}, \underline{X}) \subset \mathcal{Z}_{K}^{1}(C \underline{X}, \underline{X}) \subset \cdots \subset \mathcal{Z}_{K}^{m}(C \underline{X}, \underline{X})=\mathcal{Z}_{K}(C \underline{X}, \underline{X})
$$

is defined by

$$
\mathcal{Z}_{K}^{i}(C \underline{X}, \underline{X})=\left\{\left(x_{1}, \ldots, x_{m}\right) \in \mathcal{Z}_{K}(C \underline{X}, \underline{X}) \mid \text { at least } m-i \text { of } x_{i} \text { are basepoints }\right\} .
$$

In [7], the fat wedge filtration is shown to be quite useful in studying the homotopy type of a polyhedral product $\mathcal{Z}_{K}(C \underline{X}, \underline{X})$. For example, it is shown that the fat wedge filtration splits after a suspension so that we can recover the homotopy decomposition of Bahri, Bendersky, Cohen and Gitler [2] as follows. Let $|L|$ denote the geometric realization of a simplicial complex $L$, and put $\widehat{X}^{I}:=\bigwedge_{i \in I} X_{i}$ for a sequence of pointed spaces $\underline{X}=\left\{X_{i}\right\}_{i \in[m]}$. 
Theorem 3.1 (Iriye and Kishimoto [7] (cf. Bahri, Bendersky, Cohen and Gitler [2])). There is a homotopy decomposition

$$
\Sigma \mathcal{Z}_{K}(C \underline{X}, \underline{X}) \simeq \Sigma \bigvee_{i=1}^{m} \mathcal{Z}_{K}^{i}(C \underline{X}, \underline{X}) / \mathcal{Z}_{K}^{i-1}(C \underline{X}, \underline{X})=\Sigma \bigvee_{\emptyset \neq I \subset[m]}\left|\Sigma K_{I}\right| \wedge \widehat{X}^{I} .
$$

We call this homotopy decomposition the BBCG decomposition. Let us consider a desuspension of the BBCG decomposition. As for the moment-angle complexes, desuspension is completely characterized as:

Theorem 3.2 (Iriye and Kishimoto [7]). The moment-angle complex $\mathcal{Z}_{K}$ is a suspension if and only if its BBCG decomposition desuspends.

Then as we will see in Corollary 3.7 below that a desuspension of the BBCG decomposition of $\mathcal{Z}_{K}(C \underline{X}, \underline{X})$ is closely related with Golodness of $\mathbb{k}[K]$. So we recall from [7] a criterion for desuspending the BBCG decomposition. It is shown in [7] that to investigate the fat wedge filtration of $\mathcal{Z}_{K}(C \underline{X}, \underline{X})$, the fat wedge filtration of the real moment-angle complex $\mathbb{R} \mathcal{Z}_{K}$ plays an important role. The fat wedge filtration of $\mathbb{R} \mathcal{Z}_{K}$

$$
*=\mathbb{R} \mathcal{Z}_{K}^{0} \subset \mathbb{R} \mathcal{Z}_{K}^{1} \subset \cdots \subset \mathbb{R} \mathcal{Z}_{K}^{m-1} \subset \mathbb{R} \mathcal{Z}_{K}^{m}=\mathbb{R} \mathcal{Z}_{K}
$$

has the following property.

Theorem 3.3 (Iriye and Kishimoto [7]). There is a map $\varphi_{K_{I}}:\left|K_{I}\right| \rightarrow \mathbb{R} \mathcal{Z}_{K}^{i-1}$ for each $I \subset[m]$ with $|I|=i$ such that $\mathbb{R Z}_{K}^{i}$ is obtained from $\mathbb{R} \mathcal{Z}_{K}^{i-1}$ by attaching cones by maps $\varphi_{K_{I}}$ for all $I \subset[m]$ with $|I|=i$.

We say that the fat wedge filtration of $\mathbb{R} \mathcal{Z}_{K}$ is trivial if $\varphi_{K_{I}}$ is null homotopic for any $\emptyset \neq I \subset[m]$. Then if the fat wedge filtration of $\mathbb{R} \mathcal{Z}_{K}$ is trivial, the BBCG decomposition for $\mathbb{R} \mathcal{Z}_{K}$ desuspends. Moreover, we have:

Theorem 3.4 (Iriye and Kishimoto [7]). If the fat wedge filtration of $\mathbb{R Z}_{K}$ is trivial, then the $B B C G$ decomposition of $\mathcal{Z}_{K}(C \underline{X}, \underline{X})$ desuspends for any $\underline{X}$.

We will prove the triviality of the fat wedge filtration of $\mathbb{R} \mathcal{Z}_{K}$ for a certain simplicial complex $K$ in the next section. To this end, we will only use the following property of the maps $\varphi_{K_{I}}$ which can be seen easily from the definition of $\varphi_{K_{I}}$ in [7].

Lemma 3.5. Let $L$ be a subcomplex of $K$ on the same vertex set $[m]$. Then for any $\emptyset \neq I \subset[m]$, there is a commutative diagram

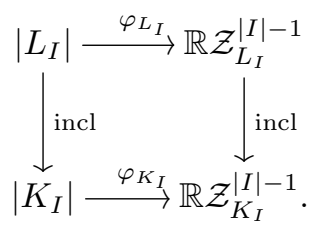

We pass to the connection between Golodness and moment-angle complexes. In [3], Baskakov, Buchstaber and Panov observed that the cellular cochain complex with coefficients $\mathbb{k}$ of the natural cell structure of the moment-angle complex $\mathcal{Z}_{K}$ is isomorphic to the Koszul resolution of $\mathbb{k}$ over $\mathbb{k}[K]$ tensored with $\mathbb{k}[K]$. As a result, we have: 
Theorem 3.6 (Baskakov, Buchstaber and Panov [3]). There is an isomorphism

$$
H^{*}\left(\mathcal{Z}_{K} ; \mathbb{k}\right) \cong \operatorname{Tor}_{\mathbb{k}\left[v_{1}, \ldots, v_{m}\right]}^{*}(\mathbb{k}[K], \mathbb{k})
$$

which respects all products and (higher) Massey products.

Since the cohomology of a suspension has trivial products and (higher) Massey products, we have:

Corollary 3.7. If $\mathcal{Z}_{K}$ is a suspension, $\mathbb{k}[K]$ is Golod for any commutative ring $\mathbb{k}$.

Then by Theorem 3.2 and 3.4, we obtain:

Corollary 3.8. If the fat wedge filtration of $\mathbb{R} \mathcal{Z}_{K}$ is trivial, then $\mathbb{k}[K]$ is Golod over any commutative ring $\mathbb{k}$.

We close this section by proving Lemma 2.3.

Proof of Lemma 2.3. By definition, we have $\mathcal{Z}_{\partial \Delta W} \cong S^{2|W|-1}$ for a finite set $W$, and $\mathcal{Z}_{K * L}=\mathcal{Z}_{K} \times \mathcal{Z}_{L}$. Then we have $\mathcal{Z}_{\partial \Delta^{I} * \partial \Delta^{J}} \cong S^{2|I|-1} \times S^{2|J|-1}$. On the other hand, $\mathcal{Z}_{K_{I}}$ is a retract of $\mathcal{Z}_{K}$. So if $K_{I \cup J}=\partial \Delta^{I} * \partial \Delta^{J}$, the cohomology of $\mathcal{Z}_{K}$ with any coefficients has a non-trivial product, implying that $K$ is not Golod by Theorem 3.6. Thus the proof is completed.

\section{Proof of Theorem 1.3}

We first investigate properties of simplicial complexes whose Stanley-Reisner rings have minimal Taylor resolutions. Then by Proposition 2.2, we consider a simplicial complex $K(\mathrm{~N})$ in Section 2. We recall notation for $K(\mathrm{~N})$. $\mathrm{N}$ is a sequence $\left\{N_{1}, \ldots, N_{r}\right\}$ of subsets of a finite set $W$, and $\widetilde{N}_{1}, \ldots, \widetilde{N}_{r}$ are minimal non-faces of $K(\mathrm{~N})$ such that $\widetilde{N}_{i}=N_{i} \sqcup\left\{a_{i}\right\}$ and $W \sqcup\left\{a_{1}, \ldots, a_{r}\right\}$ is the vertex set of $K(\mathrm{~N})$. Put $m:=|W|+r$ which is the number of vertices of $K(\mathrm{~N})$. For $w \in W$ we set

$$
\mathrm{N}_{w}:=\left\{N_{i}-w \mid i=1, \ldots, r\right\}, \quad \widehat{\mathrm{N}}_{w}:=\left\{N_{i} \mid w \notin N_{i}\right\}, \quad A_{w}:=\left\{a_{i} \mid w \in N_{i}\right\},
$$

where the ground sets of both $\mathrm{N}_{w}$ and $\widehat{\mathrm{N}}_{w}$ are $W-w$. Let $\mathrm{dl}_{K}(v)$ denote the deletion of a vertex $v$ in $K$. The following properties of the link and the deletion of $K(\mathrm{~N})$ are immediate from Lemma 2.4.

Lemma 4.1. For $w \in W$ we have

$$
\mathrm{lk}_{K(\mathrm{~N})}(w)=K\left(\mathrm{~N}_{w}\right), \quad \mathrm{dl}_{K(\mathrm{~N})}(w)=K\left(\widehat{\mathrm{N}}_{w}\right) * \Delta^{A_{w}} .
$$

We next describe the homotopy type of $|K(\mathrm{~N})|$. Let $N_{1}, \ldots, N_{r}$ be minimal nonfaces of $K$ and $L$ be a simplicial complex on the vertex set $[m]$ with minimal non-faces $M_{1}, \ldots, M_{r}$ such that $M_{i} \subset N_{i}$ for $i=1, \ldots, r$. Then $L$ is a subcomplex of $K$. Indeed, if $\sigma \subset[m]$ is a simplex of $L, M_{i} \not \subset \sigma$ for any $i$, implying $N_{i} \not \subset \sigma$ for any $i$. Then $\sigma$ is a simplex of $K$.

Proposition 4.2. We have

$$
|K(\mathrm{~N})| \simeq \begin{cases}S^{|W|-1} & N_{1} \cup \cdots \cup N_{r}=W, \\ * & \text { otherwise, }\end{cases}
$$

where we put $S^{-1}=\emptyset$. Moreover, for a sequence $\mathrm{M}=\left\{M_{1}, \ldots, M_{r}\right\}$ of subsets of $W$ 
satisfying $M_{i} \subset N_{i}$ for all $i$ and $M_{1} \cup \cdots \cup M_{r}=W$, the inclusion $|K(\mathrm{M})| \rightarrow|K(\mathrm{~N})|$ is a homotopy equivalence.

Proof. We induct on $|W|$ to get the homotopy type of $K(\mathrm{~N})$. When $|W|=0$, there is nothing to do. When $|W|=1$, we may assume $N_{1}=\cdots=N_{s}=W$ and $N_{s+1}=$ $\cdots=N_{r}=\emptyset$ for some $0 \leqslant s \leqslant r$, so

$$
K(\mathrm{~N})=W \sqcup \Delta^{\left\{a_{1}, \ldots, a_{s}\right\}} .
$$

Hence if $s \geqslant 1$, or equivalently $N_{1} \cup \cdots \cup N_{r}=W$, then $|K(\mathrm{~N})| \simeq S^{0}$, and if $s=0$, or equivalently $N_{1} \cup \cdots \cup N_{r} \neq W$, then $|K(\mathrm{~N})|$ is contractible. We assume the case $m-1$ and prove the case $m$. Notice that for any $w \in W$, there is a pushout of spaces

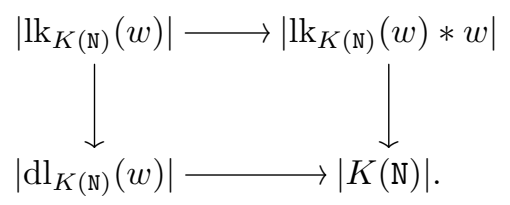

For $W \neq N_{1} \cup \cdots \cup N_{r}$, we take $w \in W-N_{1} \cup \cdots \cup N_{r}$. Then we have $\mathrm{N}_{w}=\widehat{\mathrm{N}}_{w}$, implying $\operatorname{lk}_{K(\mathrm{~N})}(w)=\mathrm{dl}_{K(\mathrm{~N})}(w)$ by Lemma 4.1 . Then we get $|K(\mathrm{~N})|=\left|\mathrm{lk}_{K(\mathrm{~N})}(w) * w\right|$ which is contractible. For $W=N_{1} \cup \cdots \cup N_{r}$, we take any $w \in W$, and we have $A_{w} \neq \emptyset$, so by Lemma $4.1\left|\mathrm{dl}_{K(\mathrm{~N})}(w)\right|$ is contractible. Since $\left|\mathrm{lk}_{K(\mathrm{~N})}(w) * w\right|$ is also contractible, we obtain $|K(\mathrm{~N})| \simeq \Sigma\left|\mathrm{k}_{K(\mathrm{~N})}(w)\right|$. By Lemma 4.1, we have $\operatorname{lk}_{K(\mathrm{~N})}(w)=$ $K\left(\mathrm{~N}_{w}\right)$ to which we can apply the induction hypothesis since the ground set of $\mathrm{N}_{w}$ is $W-w$. Thus since $N_{1} \cup \cdots \cup N_{r}=W$ if and only if $\left(N_{1}-w\right) \cup \cdots \cup\left(N_{r}-w\right)=$ $W-w$, we obtain the desired result.

We next prove the second assertion also by induction on $|W|$. The case $|W|=1$ follows from the identity (4.1). Note that the diagram (4.2) is natural with respect to the canonical inclusions between $\mathrm{M}, \mathrm{N}$. Then the second assertion holds by the induction hypothesis as above.

We next consider the fat wedge filtration of the real moment-angle complex $\mathbb{R} \mathcal{Z}_{K(\mathbb{N})}$. We prove the following simple lemma that we are going to use.

Lemma 4.3. For non-empty finite sets $A_{1}, \ldots, A_{r}$, the following hold:

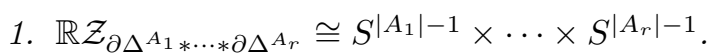

2. Let $T$ be the fat wedge of $S^{\left|A_{1}\right|-1}, \ldots, S^{\left|A_{r}\right|-1}$, that is,

$$
T:=\left\{\left(x_{1}, \ldots, x_{r}\right) \in S^{\left|A_{1}\right|-1} \times \cdots \times S^{\left|A_{r}\right|-1} \mid x_{i} \text { is the basepoint for some } i\right\} .
$$

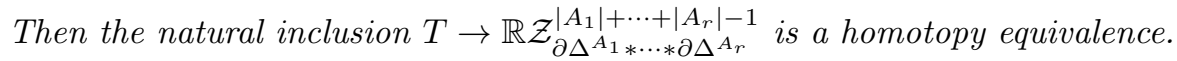

Proof. (1) In general, we have $\mathbb{R} \mathcal{Z}_{K * L}=\mathbb{R} \mathcal{Z}_{K} \times \mathbb{R} \mathcal{Z}_{L}$ for simplicial complexes $K, L$ and $\mathbb{R} \mathcal{Z}_{\partial \Delta[m]} \cong S^{m-1}$ as in the proof of Lemma 2.3. Thus we get the desired result.

(2) By definition $\mathbb{R} \mathcal{Z}_{\partial \Delta^{A_{i}}}^{\left|A_{i}\right|-1}$ is contractible, so there is a homotopy equivalence

$$
S^{\left|A_{1}\right|-1} \times \cdots \times{ }^{i} \times \cdots \times S^{\left|A_{r}\right|-1} \rightarrow \mathbb{R} \mathcal{Z}_{\partial \Delta^{A_{1}}} \times \cdots \times \mathbb{R} \mathcal{Z}_{\partial \Delta^{A_{i}}}^{\left|A_{i}\right|-1} \times \cdots \times \mathbb{R} \mathcal{Z}_{\partial \Delta^{A_{r}}} .
$$

Since the inclusion $\mathbb{R} \mathcal{Z}_{\partial \Delta^{A_{i}}}^{\left|A_{i}\right|-1} \rightarrow \mathbb{R}_{\partial \Delta^{A_{i}}}$ is a cofibration for each $i$ and

$$
\mathbb{R} \mathcal{Z}_{\partial \Delta^{A_{1} * \cdots * \partial \Delta^{A_{r}}}}^{\left|A_{1}\right|+\cdots+\left|A_{r}\right|-1}=\bigcup_{i=1}^{r}\left(\mathbb{R} \mathcal{Z}_{\partial \Delta^{A_{1}}} \times \cdots \times \mathbb{R} \mathcal{Z}_{\partial \Delta^{A_{i}}}^{\left|A_{i}\right|-1} \times \cdots \times \mathbb{R}_{\partial \mathcal{Z}^{A_{r}}}\right),
$$


the proof is completed by the standard homotopy pushout argument.

We now prove triviality of the map $\varphi_{K(\mathrm{~N})}:|K(\mathrm{~N})| \rightarrow \mathbb{R}_{K(\mathrm{~N})}^{m-1}$ of Theorem 3.3 when $\widetilde{N}_{i} \cap \widetilde{N}_{j} \neq \emptyset$ for any $i, j$, that is, under the condition 2 of Theorem 1.3. When $N_{1} \cup$ $\cdots \cup N_{r} \neq W, \varphi_{K(\mathrm{~N})}$ is trivial since $|K(\mathrm{~N})|$ is contractible by Proposition 4.2. Next we assume $N_{1} \cup \cdots \cup N_{r}=W$. We put

$$
\mathrm{M}=\left\{M_{1}, \ldots, M_{r}\right\} \quad \text { for } \quad M_{i}=N_{i}-\left(N_{1} \cup \cdots \cup N_{i-1}\right) .
$$

Now we have $M_{1} \cup \cdots \cup M_{r}=W$ and $M_{i} \subset N_{i}$ for all $i$. So by Proposition 4.2 the inclusion $|K(\mathrm{M})| \rightarrow|K(\mathrm{~N})|$ is a homotopy equivalence. By Lemma 3.5, there is a commutative diagram

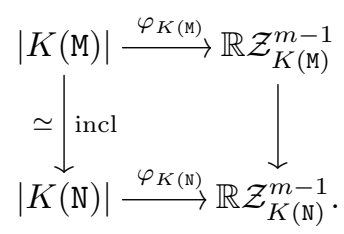

Then it is sufficient to prove that the composite around the right perimeter is null homotopic.

Since $\widetilde{M}_{i}=M_{i} \sqcup\left\{a_{i}\right\}$ and $a_{1}, \ldots, a_{r}$ are distinct, $\widetilde{M}_{i} \cap \widetilde{M}_{j}=\emptyset$ for $i \neq j$. Then we have

$$
K(\mathrm{M})=\partial \Delta^{\widetilde{M}_{1}} * \cdots * \partial \Delta^{\widetilde{M}_{r}} \text { and } K(\mathrm{M})_{U}=\partial \Delta^{\widetilde{M}_{2}} * \cdots * \partial \Delta^{\widetilde{M}_{r}}
$$

where $U=\widetilde{M}_{2} \cup \cdots \cup \widetilde{M}_{r}$ and $K(\mathrm{M})_{U}$ is the full subcomplex of $K(\mathrm{M})$ on $U$ as above. Then by Lemma 4.3 we get the following.

Proposition 4.4. We have

$$
\mathbb{R} \mathcal{Z}_{K(\mathrm{M})} \cong S^{\left|M_{1}\right|} \times \cdots \times S^{\left|M_{r}\right|}, \quad \mathbb{R} \mathcal{Z}_{K(\mathrm{M})_{U}} \cong S^{\left|M_{2}\right|} \times \cdots \times S^{\left|M_{r}\right|} .
$$

We now suppose $\widetilde{N}_{i} \cap \widetilde{N}_{j} \neq \emptyset$ for any $i, j$, and fix $2 \leqslant i \leqslant r$. By our supposition, there exists $w_{i} \in N_{1} \cap N_{i}$. Put

$$
\mathrm{M}^{i}=\left\{M_{1}^{i}, \ldots, M_{r}^{i}\right\}, \quad \text { where } \quad M_{k}^{i}= \begin{cases}M_{i} \cup w_{i} & k=i, \\ M_{k} & k \neq i .\end{cases}
$$

Then $\widetilde{M}_{j}^{i} \cap \widetilde{M}_{k}^{i}=\emptyset$ for $j \neq k$ with $j, k \geqslant 2$, so in a manner similar to Proposition 4.4 we have

$$
\mathbb{R} \mathcal{Z}_{K\left(M^{i}\right)_{U \cup w_{i}}} \cong S^{\left|M_{2}^{i}\right|} \times \cdots \times S^{\left|M_{r}^{i}\right|}=S^{\left|M_{2}\right|} \times \cdots \times S^{\left|M_{i}\right|+1} \times \cdots \times S^{\left|M_{r}\right|} .
$$

Hence the inclusion $\mathbb{R} \mathcal{Z}_{K(\mathrm{M})_{U}} \rightarrow \mathbb{R} \mathcal{Z}_{K\left(\mathrm{M}^{i}\right)_{U \cup w_{i}}}$ is identified with the inclusion $S^{\left|M_{2}\right|} \times \cdots \times S^{\left|M_{i}\right|} \times \cdots \times S^{\left|M_{r}\right|} \stackrel{1 \times \cdots \times \text { incl } \times \cdots \times 1}{\longrightarrow} S^{\left|M_{2}\right|} \times \cdots \times S^{\left|M_{i}\right|+1} \times \cdots \times S^{\left|M_{r}\right|}$ in which the $i^{\text {th }}$ coordinate sphere contracts up to homotopy. It follows that the inclu-

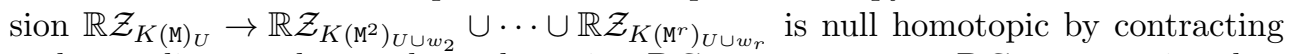
each coordinate sphere, where the union $\mathbb{R}_{K\left(\mathbb{M}^{2}\right)_{U \cup w_{2}}} \cup \cdots \cup \mathbb{R} \mathcal{Z}_{K\left(\mathbb{M}^{r}\right)_{U \cup w_{r}}}$ is taken in $\mathbb{R Z}_{K(\mathrm{~N})}^{m-1}$. Thus we obtain:

Proposition 4.5. If $\tilde{N}_{i} \cap \widetilde{N}_{j} \neq \emptyset$ for any $i, j$, then the inclusion $\mathbb{R} \mathcal{Z}_{K(\mathrm{M})_{U}} \rightarrow \mathbb{R} \mathcal{Z}_{K(\mathrm{~N})}^{m-1}$ is null homotopic. 
By Proposition 4.4 , we have $\mathbb{R} \mathcal{Z}_{K(\mathrm{M})_{U}} \cong S^{\left|M_{2}\right|} \times \cdots \times S^{\left|M_{r}\right|}$ which we abbreviate by $P$, and let $T$ be the fat wedge of $S^{\left|M_{1}\right|}, \ldots, S^{\left|M_{r}\right|}$. Then by Lemma $4.3, T \rightarrow$ $\mathbb{R} \mathcal{Z}_{K(\mathrm{M})}^{m-1}$ is a homotopy equivalence. We will show:

Lemma 4.6. The composite

$$
\Phi:|K(\mathrm{M})| \stackrel{\varphi_{K(\mathrm{M})}}{\longrightarrow} \mathbb{R} \mathcal{Z}_{K(\mathrm{M})}^{m-1} \simeq T \stackrel{\text { incl }}{\longrightarrow} T \cup C P
$$

is null homotopic.

Proof. By Theorem 3.3, the composite $|K(\mathrm{M})| \stackrel{\varphi_{K(\mathrm{M})}}{\longrightarrow} \mathbb{R} \mathcal{Z}_{K(\mathrm{M})}^{m-1} \stackrel{\text { incl }}{\longrightarrow} \mathbb{R} \mathcal{Z}_{K(\mathrm{M})}$ is a cofiber sequence. Then the composite

$$
|K(\mathrm{M})| \stackrel{\Phi}{\rightarrow} T \cup C P \stackrel{\text { incl }}{\longrightarrow} \mathbb{R} \mathcal{Z}_{K(\mathrm{M})} \cup C P
$$

is a homotopy cofiber sequence. Let $T^{\prime} \subset P$ be the fat wedge of $S^{\left|M_{2}\right|}, \ldots, S^{\left|M_{r}\right|}$. Then we have $T=\left(S^{\left|M_{1}\right|} \times T^{\prime}\right) \cup(* \times P)$, so

$$
T / P=\left(S^{\left|M_{1}\right|} \times T^{\prime}\right) /\left(* \times T^{\prime}\right)=S^{\left|M_{1}\right|} \wedge\left(T^{\prime} \sqcup *\right) .
$$

Since $T^{\prime} \sqcup *$ is a retractile subcomplex of $P \sqcup *$ in the sense of James [9], the inclusion $T^{\prime} \sqcup * \rightarrow P \sqcup *$ has a left homotopy inverse after a suspension. Thus the map

$$
\begin{aligned}
T \cup C P \simeq T / P=S^{\left|M_{1}\right|} & \wedge \\
& \left(T^{\prime} \sqcup *\right) \\
& \rightarrow S^{\left|M_{1}\right|} \wedge(P \sqcup *)=\left(S^{\left|M_{1}\right|} \times P\right) /(* \times P) \simeq \mathbb{R} \mathcal{Z}_{K(\mathrm{M})} \cup C P
\end{aligned}
$$

has a left homotopy inverse since $\left|M_{1}\right| \geqslant 1$, where this composite is homotopic to the second map of the homotopy cofiber sequence (4.4). Thus the map $\Phi:|K(\mathrm{M})| \rightarrow$ $T \cup C P$ is null homotopic as desired, completing the proof.

By Proposition 4.5, the inclusion $P \rightarrow \mathbb{R} \mathcal{Z}_{K(\mathrm{~N})}^{m-1}$ is null homotopic. Then the map $T \rightarrow \mathbb{R}_{K(\mathrm{~N})}^{m-1}$ extends to a map $T \cup C P \rightarrow \mathbb{R}_{K(\mathrm{~N})}^{m-1}$, and by Theorem 3.3, the commutative diagram (4.3) extends to a homotopy commutative diagram

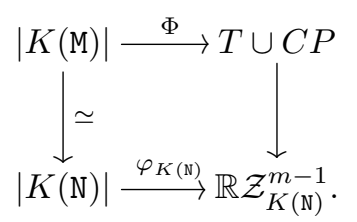

Thus the map $\varphi_{K(\mathrm{~N})}$ is null homotopic by Lemma 4.6, and therefore we have established the following.

Proposition 4.7. If $\widetilde{N}_{i} \cap \widetilde{N}_{j} \neq \emptyset$ for any $i, j$, the map $\varphi_{K(\mathbb{N})}$ is null homotopic.

Theorem 4.8. If $\widetilde{N}_{i} \cap \widetilde{N}_{j} \neq \emptyset$ for any $i, j$, then the fat wedge filtration of $\mathbb{R}_{K(\mathrm{~N})}$ is trivial.

Proof. Suppose $\widetilde{N}_{i} \cap \widetilde{N}_{j} \neq \emptyset$ for any $i, j$. By Proposition 4.7, it is sufficient to prove that every full subcomplex of $K(\mathrm{~N})$ is either contractible or isomorphic to $K(\mathrm{M})$ for some M. Since every full subcomplex is obtained by deleting vertices, the following claim proves the statement above.

Claim: For any vertex $v$ of $K(\mathrm{~N}), \mathrm{dl}_{K(\mathrm{~N})}(v)=K(\mathrm{M}) * \Delta^{S}$ for some $S, \mathrm{M}$ such that any two elements of $\mathrm{M}$ are not disjoint, where $\mathrm{M}, S$ may be empty.

We divide the proof of the claim into the following two cases: 
Case $v \in W$ : By Lemma 4.1, $\mathrm{dl}_{K(\mathrm{M})}(v)=K\left(\widehat{\mathrm{N}}_{v}\right) * \Delta^{A_{v}}$. By our supposition, any two elements of $\widehat{\mathrm{N}}_{v}$ are not disjoint. Then the claim is true for $K\left(\widehat{\mathrm{N}}_{v}\right) * \Delta^{A_{v}}$.

Case $v \notin W$ : Since $v=a_{i}$ for some $i$, we have $\mathrm{dl}_{K(\mathrm{~N})}(v)=K(\mathrm{M})$, where $\mathrm{M}=\left\{N_{j} \mid\right.$ $j \neq i$. Then the claim is obviously true for $K(\mathrm{M})$.

Proof of Theorem 1.3. The implication $1 \Rightarrow 2$ follows from Proposition 2.5. If 2 holds, then by Proposition 2.2 and Theorem 4.8, the fat wedge filtration of $\mathbb{R} \mathcal{Z}_{K}$ is trivial. Thus by Theorem 3.4, 4 holds. Moreover, by Proposition 4.2, 3 holds. When 3 or 4 holds, 1 holds by Corollary 3.7. Therefore the proof is completed.

\section{References}

[1] A. Ayzenberg, Buchstaber numbers and classical invariants of simplicial complexes, arXiv:1402.3663.

[2] A. Bahri, M. Bendersky, F.R. Cohen, and S. Gitler, The polyhedral product functor: a method of decomposition for moment-angle complexes, arrangements and related spaces, Adv. Math. 225 (2010), 1634-1668.

[3] I.V. Baskakov, V.M. Buchstaber, and T.E. Panov, Cellular cochain algebras and torus actions, Russian Math. Surveys 59 (2004), no. 3, 562-563.

[4] M.W. Davis and T. Januszkiewicz, Convex polytopes, Coxeter orbifolds and torus actions, Duke Math. J. 62 (1991), 417-452.

[5] R. Frankhuizen, $A_{\infty}$-resolutions and the Golod property for monomial rings, arXiv: 1612.02737.

[6] E.S. Golod, On the homologies of certain local rings, Soviet Math. Dokl. 3 (1962), 745-748.

[7] K. Iriye and D. Kishimoto, Fat wedge filtrations and decomposition of polyhedral products, to appear in Kyoto J. Math.

[8] K. Iriye and D. Kishimoto, Golodness and polyhedral products for two dimensional simplicial complexes, to appear in Forum Math. https://doi.org/10. 1515/forum-2017-0130

[9] I.M. James, On H-spaces and their homotopy groups, Q. J. Math. 11 (1960), 161-179.

[10] L. Katthän, The Golod property for Staney-Reisner rings in varying characteristic, J. Pure Appl. Algebra 220 (2016), 2265-2276.

[11] L. Katthän, A non-Golod ring with a trivial product on its Koszul homology, J. Algebra 479 (2017), 244-262.

Kouyemon Iriye kiriye@mi.s.osakafu-u.ac.jp

Department of Mathematics and Information Sciences, Osaka Prefecture University, Sakai, 599-8531, Japan

Daisuke Kishimoto kishi@math.kyoto-u.ac.jp

Department of Mathematics, Kyoto University, Kyoto, 606-8502, Japan 